ORIGINAL ARTICLE

\title{
Retinopathy and Neuropathy in Patients with End Stage Renal Disease on Haemodialysis- A Hospital Based Study
}

\author{
*N Mahmood ${ }^{1}$, SS Chowdhury ${ }^{2}$ \\ 1*Dr. Nazneen Mahmood, Associate Professor \& Head of Nephrology, AKMMC.nazneendr31@gmail.com \\ ${ }^{2}$ Dr. Shaila Safia Chowdhury, Assistant Professor, Department of Medicine, Medical College for Women and Hospital \\ *Corresponding Author
}

\begin{abstract}
Bacground: Chronic renal failure is irreversible and progressive process that results in end stage renal disease (ESRD) where the patient has to be dependent on renal replacement therapy for survival. Retinopathy and neuropathy are often asymptomatic in their most treatable stage; delay in diagnosis can result in significant increase in the patient's risk of visual loss and disability. Ocular condition is also an indicator of the metabolic control of the disease process. This study is an attempt to access the ocular status/complications as well as neurological manifestation associated with end stage renal disease on haemodialysis.
\end{abstract}

Methodology: This is a cross sectional descriptive type of observational study on patients of end stage renal disease getting haemodialysis in Medical College for Women and Hospital. Duration of study was 3 years from January 2011 to December 2013. This study is an attempt to access the ocular status/complications as well as neurological manifestation associated with end stage renal disease on haemodialysis.

Result: Among 81 patients, 42 patients had hypertension (HTN) and 19 had diabetes mellitus (DM) and 20 had other causes of chronic kidney disease(CKD). In our study, out of 19 patients of diabetes mellitus, $73.68 \%(14)$ had diabetic retinopathy(DR) and $26.32 \%(5)$ had normal fundus. All the 42 patients had hypertensive retinopathy(HR). Regarding fundal examination of patients with hypertensive retinopathy(HR), $32.26 \%$ had gradel, and $17.74 \%$, $43.55 \%$ and $6.45 \%$ were of grade $11,111,1 \mathrm{~V}$ respectively. It was found that non-proliferative changes in ocular fundal examination in diabetic patients were the commonest abnormality. On fundal examination of diabetic retinopathy (DR), 26.32\% had normal fundus, 26.32\% had dot and blot haemorrhages, $21.05 \%$ had hard exudates and cotton wool, $21.05 \%$ had new vascularization while $5.26 \%$ had retinal detachment.The following types of neuropathy were seen among our patients; $48.15 \%$ had symmetrical distal sensory motor polyneuropathy, $19.75 \%$ had a symmetrical polyneuropathy, $19.75 \%$ had mononeuropathy and cranial nerve palsies were detected in $12.35 \%$.

Conclusion: Detailed ocular and neurological examination should be undertaken in patients of end stage renal disease(ESRD) on maintenance haemodialysis(HD) for early diagnosis and treatment of the complications. Awareness is needed of the potential ocular and neurological complications of the disease process.

Key Words: Retinopathy \& Neuropathy in end stage renal disease(ESRD), Haemodialysis' Hypertensive retinopathy, Diabetic retinopath

\section{Introduction}

Chronic renal failure is irreversible and progressive process that results in end stage renal disease (ESRD) where the patients has to be dependent on renal replacement therapy for survival ${ }^{1}$. The worldwide dialysis population is estimated to be greater than 1.1 million persons ${ }^{2}$. Compared to Japan, Taiwan and United States most countries have a lower incidence of treated end stage renal disease (ESRD) ${ }^{2}$. Haemodialysis is the predominant technique for treating end stage renal disease (ESRD) throughout the world.

AKMMC J 2015; 6(1): 14-19
Approximately 100-120 patients per million population (pmp) reaches end stage renal disease (ESRD) every year in Bangladesh, which is not very different what is being reported from countries in Asia and Middle East $^{3}$. So end stage renal disease (ESRD) is a global problem, which should encountered with definite strategy. Our inclusion criteria to elicit patients were as follows- receiving three months dialysis, twice weekly at least, having $>8$ years old and medication stability, using a native arterio-venous fistula in the cubital or radial area as vascular access. Any history of upper limb deformity or fracture make the patients excluded from the study. 
Richard Bright, 1836 first accounted renal disease with blindness. Later on it was recognized that uremic retinitis is the manifestation of hypertension $(\mathrm{HTN})^{4}$. Deterioration of eyesight is due to worsening of hypertension or diabetic retinopathy, ischemic optic neuropathy, central retinal vein occlusion and cortical blindness. By the end stage renal disease (ESRD), $80.0 \%$ of patients will have developed secondary hypertension $(\mathrm{HTN})^{5}$. Ocular morbidity may be directly due to hypertension (HTN), uremia and anaemia; some are related to the causes leading to chronic renal failure. Some effects are due to haemodialysis.

Hypertensive retinopathic changes are particularly severe in renal failure. This has been attributed to the effects of retained nitrogen products ${ }^{6}$. The ophthalmic appearance is of value in determining the efficacy of the anti-hypertensive therapy ${ }^{7}$. Diabetic retinopathy(DR) tends to deteriorate with falling renal function, poorly controlled blood pressure $^{8,9}$. Diabetes mellitus (DM) can affect the eye in a number of ways, the most common and characteristic form is diabetic retinopathy which is subdivided into three stages: background retinopathy, pre-proliferative retinopathy and proliferative retinopathy ${ }^{10,11}$. Uremic neuropathy highly limits the individual self- sufficiency causing near-continuous pain. The following varieties of neuropathy may occur: symmetrical mainly sensory polyneuropathy, acute painful neuropathy, mononueropathy and mononeuritis multiplex ${ }^{12}$.

Retinopathy and neuropathy are often asymptomatic in their most treatable stage; delay in diagnosis can result in significant increase in the patient's risk of visual loss and disability. Ocular condition is also an indicator of the metabolic control of the disease process. This study is an attempt to access the ocular status/complications as well as neurological manifestation associated with end stage renal disease on haemodialysis.

\section{Materials and Methods}

This is a cross sectional descriptive type of observational study on patients of End stage renal disease getting haemodialysis in Medical College for Women and Hospital. Duration of study was 3 years from January 2011 to December 2013. Our inclusion criteria to elicit patients were as follows- receiving three months dialysis, twice weekly at least, having $>$ 18 years old and medication stability, using a native arterio-venous fistula in the cubital or radial area as vascular access. Any history of upper limb deformity or fracture make the patients excluded from the study. The grading of hypertensive retinopathy presented in Table-1.

A standard bicarbonate solution was used for dialysis. Polysulphone low flux dialysis membrane ( Fresenius F- series Fresenius Medical Care, Bad Homburg, Germany) were used for all the dialysis sessions. Only dialysis patients with $\mathrm{Kt} / \mathrm{V}>1.0$ (Daugirdas formula) were included. Hypertensive patients were on anti hypertensive therapy. In all subjects both blood pressure ( $\mathrm{BPCi}$ ) and heart rate ( $\mathrm{HRCi}$ ) change ability indexes. The study protocol was approved by the local Ethical committee. Written informed consent was obtained from all the patients. A questionnaire was elaborated and filled for each by the author.

The questionnaire consisted of the following: Clinical history, clinical examination was divided into general and systemic examination and detailed neurological examination emphasizing on the cranial nerves, upper and lower limb examination in addition to autonomic nervous system examination. Dilated fundal examination using medriacyl, phenylephrine to evaluate the posterior segment and the retina was done by the author and re-evaluated by ophthalmologist. Then retinopathy was staged into- mild nonproliferative, moderate non-proliferative, severe nonproliferative and proliferative diabetic retinopathy. Neuropathy was diagnosed when the patients complained of burning or loss of sensation, pain or tingling in addition to impaired or absent tendon reflexes, decreased sensitivity to light touch and pin prick, impaired vibration and position senses. The information on dialysis blood pressure, inadequacies or orthostatic faints, presence of itching of skin, constipation and diarrhea, feeling of fullness of stomach and sweating during eating were included. The presence of intra-dialytic hypotension was defined as a fall of systolic blood pressure to less than $90 \mathrm{~mm}$ of $\mathrm{Hg}$ or more than $20 \mathrm{~mm}$ of $\mathrm{Hg}$ from the pre- dialysis value in at least $50 \%$ of dialysis session. A four stage patient evaluation during intense breathing, static effort, valsalva maneuver after assuming erect position. Before an evaluation the participants were asked to refrain from smoking and drinking strong tea or coffee and not to have heavy meals for 12 hours before the tests. The tests were always performed at the end of mid-week haemodialysis session. In the study group $\mathrm{Kt} / \mathrm{V}$ values and percentage body mass increases per haemodialysis (calculated as weight before dialysis- dry body weight/dry body weight $\mathrm{x} 100 \%$ ) showed no statistically significant differences among participants. 
The following investigations were done for each patients- Complete blood count, Fasting blood sugar, Blood sugar 2 hours after breakfast, Serum creatinine, Urine $\mathrm{R} / \mathrm{E}$ (routine examinatio), Urine for microalbuminuria and macroalbuminuria, lipid profile, electrocardiogram(ECG), ultrasonogram(USG) of whole abdomen, $\mathrm{S}$ electrolytes, $\mathrm{S}$ Calcium, S Phosphate, $\mathrm{HbA1C}$ was measured to asses the degree of metabolic control. Data were introduced into the computer from a master sheet recording using software programme. Data entered and analyzed, then the results expressed in the form of table, figures and graphs using Statistical Package for Social Science (SPSS- Version 15) programme. Results were expressed as Mean Standard Deviation for continuous data and as frequency with percentages for categorical data.

\section{Results}

Out of 81 patients 48 were males and 33 were females, with male to female ratio of 1.5:1.The mean age of the patient was 50.8112 .45 ( Range-22-75 )year. Age distribution was found as follows: 20-29 years represents $4.95 \%, 30-39$ years represents $8.64 \%$, 40-49 years represents $24.69 \%, 50-59$ years represents $29.63 \%$, 60-69 years represents $23.46 \%$ and $70-79$ years represents $8.63 \%$ (Table-2). Among 81 patients, 42 patients had hypertension(HTN) and 19 had diabetes mellitus(DM) and 20 had other causes of chronic kidney disease(CKD). The duration of DM among the studied group ranged between 5-24 years and HTN between 2-20 years. It did appear that most of the patient had associated systemic diseases. 43 patients had ischemic heart disease(IHD) and 75 patients suffered from dyslipidaemia(Table-3).

The common presenting ocular symptom among patients of end stage renal disease(ESRD) was found to be blurring of vision(70.37\%), floater in $13.58 \%, 4$ patients $(4.94 \%)$ present with loss of vision while $11.11 \%$ had no symptoms(Table-4). Numbness was detected in $11.11 \%$ of our patient, parasthesia in $13.58 \%, 59.26 \%$ presented with burning sensations, $6.17 \%$ with weakness and $9.88 \%$ with no symptoms (Table-5).

In our study, out of 19 patients of diabetes mellitus, $73.68 \%(14)$ had diabetic retinopathy(DR) and $26.32 \%(5)$ had normal fundus. All the 42 patients had hypertensive retinopathy(HR). Regarding fundal examination of patients with hypertensive retinopathy, $32.26 \%$ had grade 1 , and $17.74 \%, 43.55 \%$ and $6.45 \%$ were of grade $11,111,1 \mathrm{~V}$ respectively (Table-6). It was found that non-proliferative changes in ocular fundal examination in diabetic patients were the commonest abnormality. On fundal examination of diabetic retinopathy, $26.32 \%$ had normal fundus, $26.32 \%$ had dot and blot haemorrhages, $21.05 \%$ had hard exudates and cotton wool, $21.05 \%$ had new vascularization while $5.26 \%$ had retinal detachment (Table-7).

Concerning examination of upper and lower limbs $92.60 \%$ had muscle power grade-5, $1.23 \%$ had wasting, $2.47 \%$ had power grade- $4,2.47 \%$ had power grade- 3 and $1.23 \%$ had power grade-2 (Table- 8 ). The study showed varieties of sensory disturbance, $93.82 \%$ presented with decrease vibration sense, $51.85 \%$ with decrease pin prick sensation and $77.78 \%$ had loss of position sense (Table-9). The following types of neuropathy were seen among our patients; $48.15 \%$ had symmetrical distal sensory motor polyneuropathy, $19.75 \%$ had a symmetrical polyneuropathy, $19.75 \%$ had mononeuropathy and cranial nerve palsies were detected in $12.35 \%$ (Table-10).

The mean serum creatinine was $8.51-1.54 \mathrm{mg} / \mathrm{dl}$ and mean haemoglobin concentration and random blood sugar of the study group was $8.45-1.73 \mathrm{gm} / \mathrm{dl}$ and 7.65-2.81 $\mathrm{mmol} / \mathrm{L}$ respectively.

Table-1: Grading of Hypertensive Retinopathy

Grade-I Mild arteriolar attenuation, broadening of arteriolar light reflex

Grade-II Severe arteriolar constriction associated with deflection of veins at arteriovenous crossing

Grade-III Copper wiring of arterioles, banking and tapering of veins, right-angled deflection of veins, haemorrhages, cotton-wool spots, hard exudates

Grade-IV Changes of grade III, silver wiring of arterioles, disc swelling.

Ref-22

Table-2: Distribution of Patients according to Age:

\begin{tabular}{lll}
\hline Age distribution in years & No & \% \\
\hline $20-29$ & 4 & 4.95 \\
$30-39$ & 7 & 8.64 \\
$40-49$ & 20 & 24.69 \\
$50-59$ & 24 & 29.63 \\
$60-69$ & 19 & 23.46 \\
$70-79$ & 7 & 8.63 \\
Total & $\mathbf{8 1}$ & $\mathbf{1 0 0 . 0 0}$ \\
\hline
\end{tabular}


AKMMC J $2015: 6(1)$

Table-3: Distribution of Patients according to Systemic Diseases:

\begin{tabular}{lll}
\hline Systemic Diseases & No & $\%$ \\
\hline Dyslipidaemia & 75 & 92.59 \\
IHD & 43 & 53.08 \\
HTN & 42 & 51.85 \\
DM & 19 & 23.46 \\
Other causes of CKD & 20 & 24.69 \\
\hline
\end{tabular}

Table-4: Distribution of Patients according to Ocular Symptoms

\begin{tabular}{llc}
\hline Symptoms & No & \% \\
\hline Blurring of Vision & 57 & 70.37 \\
Floater & 11 & 13.58 \\
Loss of Vision & 4 & 4.94 \\
No Symptoms & 9 & 11.11 \\
Total & $\mathbf{8 1}$ & $\mathbf{1 0 0 . 0 0}$ \\
\hline
\end{tabular}

Table-5: Distribution of Patients according to Symptoms of Nervous System

\begin{tabular}{lll}
\hline Symptoms & No & \% \\
\hline Numbness & 9 & 11.11 \\
Parasthesia & 11 & 13.58 \\
Burning Sensation & 48 & 59.26 \\
Weakness & 5 & 6.17 \\
No Symptoms & 8 & 9.88 \\
Total & $\mathbf{8 1}$ & $\mathbf{1 0 0 . 0 0}$ \\
\hline
\end{tabular}

Table-6: Distribution of Patients according to Grades of Hypertensive Retinopathy:

\begin{tabular}{llll}
\hline \multicolumn{2}{c}{ Grades } & No & \% \\
\hline Grade & - I & 10 & 23.81 \\
Grade & - II & 11 & 26.19 \\
Grade & - III & 17 & 40.48 \\
Grade & - IV & 4 & 9.52 \\
Total & & $\mathbf{4 2}$ & $\mathbf{1 0 0 . 0 0}$
\end{tabular}

Table-7: Distribution of Patients according to Diabetic Retinopathy:

\begin{tabular}{llc}
\hline Fundus Examination & No & \% \\
\hline Normal Fundus & 5 & 26.32 \\
Dot \& Blot Haemorrhage & 5 & 26.32 \\
Hard Exudate with Cotton & 4 & 21.05 \\
Wool & & \\
New vascularization & 4 & 21.05 \\
Retinal Detachment & 1 & 5.26 \\
Total & $\mathbf{1 9}$ & $\mathbf{1 0 0 . 0 0}$ \\
\hline
\end{tabular}

N Mahmood, SS Chowdhury

Table-8: Distribution of Patients according to Muscle Power:

\begin{tabular}{cll}
\hline Muscle Power & No & \% \\
\hline Wasting & 1 & 1.23 \\
Grade-2 & 1 & 1.23 \\
Grade-3 & 2 & 2.47 \\
Grade-4 & 2 & 2.47 \\
Grade-5 & 75 & 92.60 \\
Total & $\mathbf{8 1}$ & $\mathbf{1 0 0 . 0 0}$ \\
\hline
\end{tabular}

Table-9: Distribution of Patients according to Varieties of Sensory Disturbance

\begin{tabular}{lcc}
\hline Sensory Disturbance & No & $\%$ \\
\hline Decrease Vibration Sense & 76 & 93.82 \\
Decrease Pinprick Sensation & 42 & 51.85 \\
Loss of Position Sense & 63 & 77.78 \\
\hline
\end{tabular}

Table-10: Distribution of Patients according to Types of Neuropathy

\begin{tabular}{lcc}
\hline Types of Neuropathy & No & $\%$ \\
\hline $\begin{array}{l}\text { Symmetrical Distal Sensory } \\
\text { Motor Neuropathy }\end{array}$ & 39 & 48.15 \\
Symmetrical Polyneuropathy & 16 & 19.75 \\
Mononeuropathy & 16 & 19.75 \\
Cranial Nerve Palsies( III, IV, VI) & 10 & 12.35 \\
Total & $\mathbf{8 1}$ & $\mathbf{1 0 0 . 0 0}$ \\
\hline
\end{tabular}

\section{Discussion}

The study showed that male to female was found to be $1.5: 1$ which was similar to worldwide data ${ }^{1}$. Another study of L. Bajracharya et al showed that male to female ratio was $2.3: 1^{13}$. According to age distribution $60 \%$ of our patients was above 50 years and $24(29.63 \%)$ with in 50-59 years. The mean age of the patient was 50.8112 .45 ( Range-22-75 )year. In the study of L. Bajracharya et al mean age of total patients of chronic renal failure(CRF) was 48.3 14.9 years which was almost similar to our study ${ }^{13}$.

The commonest cause of end stage renal disease (ESRD) in our study was hypertension(HTN) $42(51.85 \%)$ followed by diabetes mellitus(DM) $19(23.46 \%)$. But in the study of L. Bajracharya et al $36.1 \%$ were of HTN and $27.7 \%$ and $20.2 \%$ were of $\mathrm{DM}$ and glomerulonephritis respectively ${ }^{13}$. The majority of the patients have arterial hypertension 
before starting haemodialysis(HD) treatment. After starting with maintenance $\mathrm{HD}$, the hypertension is usually more or less regulated with or without therapy ${ }^{14}$. Some patients remain hypertensive because of hyper-reninaemia. In most cases, routine antihypertensive drug therapy is effective ${ }^{14}$. Unfortunately, no exact data on duration of hypertension prior to HD treatment were obtained, so we could not compare the influence of hypertension duration on visual field loss in our HD patients. No typical type of field loss was observed. We found no similar pattern of location of visual field defects between our HD patients.

HD patients have advanced atherosclerosis in carotid arteries compaired with age and sex matched control subjects ${ }^{15}$. The intima-media thicknesses of the common carotid and internal carotid arteries were higher in HD patients compared with controls. There was a positive correlation with age, but not with duration of HD treatment ${ }^{16}$. The causes for these complications are hypertension, hyperlipidaemia, glucose intolerance, chronic high cardiac output, metastatic vascular and myocardial calcification ${ }^{15}$.

$70.37 \%$ (57) of our patient presents with blurring of vision whereas $62 \%$ patients had similar complaints in the study of L Bajracharya et $\mathrm{al}^{13}$. In another study Abbashar Hussein Mohmad et al showed that $88.2 \%$ patients of DM were suffering from blurring of vision ${ }^{17}$. So our study was almost similar to other studies.

In ESRD patients, pathologic changes could be found in many tissues and organs including the eyes. Regular and more frequent ocular examinations have been suggested in these patients ${ }^{18}$. Many patients on maintenance haemodialysis suffer from chronic eye diseases induced by both dialysis procedure and the uremic miliew ${ }^{19}$. In a study of $L$ Bajracharya et al it was shown that $48 \%$ of total patients of chronic kidney disease had hypertensive retinopathy(HR) and $88.3 \%$ of total diabetics had diabetic retinopathy(DR $)^{13}$. In our study $73.68 \%(14)$ of diabetic patients had DR and 42 patients had HR, which was similar to the study of L Bajracharya et al.

Diabetic patients are prone to visual disturbances such as decrease visual acuity and even blindness due to retinopathy. These effects are more common in diabetic haemodialysis patients ${ }^{20,21}$. In our study, out of 19 patients of diabetes mellitus, $73.68 \%(14)$ had diabetic retinopathy(DR) and $26.32 \%(5)$ had normal fundus. All the 42 patients had hypertensive
retinopathy(HR). But in the study of L Bajracharya et al hypertensive retinopathy(HR) was present in $47.1 \%$ and diabetic retinopathy(DR) was present in $88.3 \%$ of the patients, which was different from our study. ${ }^{13}$ Regarding fundal examination of our patients, $40.48 \%$ (17) patients had Grade-III hypertensive retinopathy(HR) and $26.19 \%(11)$ patients had Grade-II HR, and $23.82 \%(11)$ and 9.52\%(4) were of Grade-I and Grade-IV respectively. But in the study of D Pahor, Grade-I HR was evident in ${ }^{16}$ eyes and Grade-II in remaining 24 eyes of HD patients $^{22}$. No cases of severe HR (Grade-III and Grade-IV) were encountered in D Pahor study ${ }^{22}$. Our study was different from this. Whereas in the study of L Bajracharya et al $92 \%$ Grade-III HR and all 4 cases of Grade-IV HR were detected ${ }^{13}$ which was similar to our study. It was found that non-proliferative changes in ocular fundal examination in diabetic patients were the commonest abnormality in our study. On fundal examination of diabetic retinopathy(DR), in our study, $26.32 \%$ had normal fundus, $26.32 \%$ had dot and blot haemorrhages, $21.05 \%$ had hard exudates and cotton wool, $21.05 \%$ had new vascularization while $5.26 \%$ had retinal detachment It was similar to the study of Abbashar Hussein Mohmad et al and a study of Peshwar ${ }^{17,23}$.

In the study of Dyck PJ et al,the most common neurological complication of diabetes(DM) is the development of a distal symmetrical poly-neuropathy affecting $50 \%$ of patients at long term follow-up24. Symetrical distal sensory motor poly-neuropathy was the commonest type $(48.15 \%)$ of perepheral neuropathy in our study. This was similar to what was mentioned in the literature ${ }^{17,25,26}$. Multiple cranial nerves involvement was one of the neurological complications associated with diabetes with end stage renal disease(ESRD). The most common cranial nerves involved in our study were third, fourth and six.

\section{Conclusion}

Detailed ocular and neurological examination should be undertaken in patients of end stage renal disease(ESRD) on maintenance haemodialysis (HD) for early diagnosis and treatment of the complications. Awareness is needed of the potential ocular and neurological complications of the disease process. This was a single center study with small number of patients, further research on multiple center with longer follow-up and diverse time frames is required to confirm the findings. 


\section{Acknowledgement}

We would like to thank all the patients, personnel of haemodialysis department of Medical College for Women and Hospital, Uttara, Dhaka for their kind cooperation. I like to thank Dr. Palash Barua, Assistant Professor of Ophthalmology, MCWH, Uttara for his kind help regarding of ophthalmological examination of the patients.

\section{Conflict of interest : None.}

\section{References}

1. Weatherall DJ, Lidingham JGG, and Warrell DA. Oxford textbook of medicine Vol III. 3rd ed. OxfordNewyork- Tokyo: Oxford Univ Press 1996; 3294-5.

2. Goodman WG \& Danovitch GM. Options for patients with kidney failure. In:Handbook of Kidney Transplantation. Philadelphia: Lippincott William \&Wilkins 4th edition. 2008; 3-21.

3. Rashid HU. Bangladesh renal registry report (1986-1996) Bangladesh renal J 2002;21(1):s25-28.

4. Duke-Elders S, Dohree JH. System of Ophthalmology, Vol X. 1st ed. London: The CV Mosby Company 1967; Chapter 4, 315-47.

5. Stein $\mathrm{JH}$, Hulton JJ, Kohler $\mathrm{PO}$ et al. International Medicine. 3rd ed. USA: Little Brown \& Comp 1990;809-10.

6. Duane TD, Jaeger EA. Duane's clinical ophthalmology Vol 5. Revised ed. USA: Harper and Row 1987; Chapter 31,1-2.

7. Peyman GA, Sanders DR, Goldberg MF. Principles and Practice of Ophthalmology. New Delhi: Jaypee Brothers 1987: 1205-35, 1633-40.

8. Schmechel H, Heinrich U. Retinopathy and neuropathy in 772 insulin-treated diabetic patients in relation to the type of diabetes. Diabetes Metabol 1993; 19:138-42.

9. Janka HU, Ziegler AG, Valsania P, Warram JH, Krolewski AS. Impact of blood pressure on diabetic retinopathy. Diabetes Metabol 1989;15:333-7.

10. Rosenbloom Al, Emerging epidemic of type 2 diabetes in Youth. Diabetic Care 1999;22:345-347.

11. Gerich JE. The genetic basis of type 2 diabetes mellitus: impaired insulin secretion versus impaired insulin sensitivity. Endocr Rev 1998;19:491.

12. James M. Falko, David E. Jones. Standards of medical care in diabetes. Diabetes Care 2005;28(1):4-36.

13. L Bajracharya, DN Shah, KB Raut and S Koirala. Ocular evaluation in patients with chronic renal failurea hospital based study. Nepal Med Coll J 2008;10(4):209-214.
14. Polak BCP. Ophthalmological complications associated with haemodialysis. In: Maher JF(ed). Replacement of Renal Function by Dialysis: A Textbook of Dialysis. Kluwer Academic Publisher: Dordrecht, 1981, pp 987991.

15. Brenner BM, Lazarus JM. Chronic renal failure. In: Wilson JD(ed). Harrison's Principles of Internal Medicine. McGraw-Hill: New York, 1991, pp 11501156.

16. Hojs R. Carotid intima-media thickness and plaques in haemodialysis patients. Artif Organs 2000;24(9):691695.

17. Abbashar Hussein Mohmad, Amal Hassan, Amira Sidig Mohammed OH Gadour, Ahmad Hamad, Mohmad Malk Aldar. Correlation between Retinopathy, Nephropathy and Perepheral Neuropathy among Adult Sudanese Patients. Sudan JMS 2011; 6(1): 27-32.

18. Vrabec R, Vatavuk Z, Pavlovic D et al. Ocular findings in patients with chronic renal failure undergoing haemodialysis. Coll Antropol. 2005;29 Suppl 1:95-8.

19. Evans RD, Rosner M. Ocular abnormalities associated with advanced kidney disease and haemodialysis. Semin Dial. 2005;18: 252-7.

20. Clark A, Morgan WH, Kain S et al. Diabetic retinopathy and the major causes of vision loss in Aboriginals from remote Western Australia. Clin Experiment Ophthalmol. 2010;38:475-82.

21. de Fine ON, Siersma V, Almind GJ, Nielson NV. Prevalence and progression of visual impairment in patients newly diagnosed with clinical type 2 diabetes: a six year follow up study. BMC Public Health. 2011;11: 80.

22. D Paher. Retinal light sensitivity in haemodialysis patients. Eye. 2003;17:177-182.

23. Shafiqur Rahman, Irfan Zia. Prevalence of microvascular complications among diabetic patients. Pakistan JMed Res 2004;43(4):1-3.

24. Dyck PJ, Kratz KM, Karnes JL, Litchy WJ, Klein R, Pach JM et al. The prevalence by staged severity of various types of diabetic neuropathy, retinopathy and nephropathy in a popular based cohort: th rochester diabetic neuropathy study. Nephrology. 1993;43:81724.

25. K Adour, J Wingerd and HE Doly. Prevalence of concurrent diabetes mellitus and idiopathic facial paralysis. Diabetes. 1975;5:449-451.

26. Lazzaroni F, Laffi GL, Galuppi V et al. Paralysis of oculomotor nerves in diabetes mellitus. Rev Neurol. 1993;149:571. 